\title{
MÉXICO 2008: EL AÑO EN QUE SE VIVIÓ EN PELIGRO
}

\author{
Mexico 2008: The Year of Living Dangerously
}

\section{JOY LANGSTON}

CIDE-México

\section{BERNARDO PÉREZ}

\author{
CIDE-México
}

\section{RESUMEN}

El trabajo muestra los obstáculos que tendrá que librar la administración si acaso quiere seguir contando con el apoyo de los votantes, como el combate al narcotráfico y los efectos de la crisis financiera de EUA en la economía nacional. También analiza el paquete de reformas con las que el Presidente Calderón pretende darle continuidad a sus políticas en el 2008: la judicial, que modernizaría el sistema de impartición de justicia; la energética, que modificaría el régimen de la empresa más importante del Estado, y la educativa, que reduciría los rezagos educativos mediante maestros mejor capacitados. También discute las características del Congreso que las aprueba, entre ellas un PRD dividido, un PRI cada vez más fuerte en los estados y unas elecciones intermedias cada vez más cercanas.

Palabras clave: PEMEX, narcotráfico, reforma judicial, Felipe Calderón.

\begin{abstract}
This article explains how the fight against narco-trafficking and the economic crisis has affected the PAN's popularity at the polls, and the voters' image of the president's leadership ability. It also reviews the central reforms undertaken by president Calderón in 2008 , including the overhaul of the judicial system, a minor reform of the public energy sector, and a new set of rules to modernize public education. It then examines internal dynamics of the divided PRD and resurging electoral fortunes of the PRI in many states.
\end{abstract}

Key words: PEMEX, drug-trafficking, judicial reform, Felipe Calderón. 


\section{INTRODUCCIÓN}

El segundo año de la presidencia de Felipe Calderón fue un periodo significativamente más difícil en términos de avance de las políticas públicas; y a diferencia de sus primeros doce meses, la administración fue menos exitosa. El presidente continuó con su estrategia de utilizar al ejército para combatir al narcotráfico, lo que le permitió celebrar algunas victorias como la captura de miembros importantes de los carteles, así como la detención de autoridades gubernamentales que trabajaban con el crimen organizado. Esta estrategia, sin embargo, tuvo también consecuencias negativas, pues los traficantes respondieron de manera enérgica en contra de otros narcotraficantes y de los cuerpos policiacos y del ejército. Los narcos recurrieron a tácticas menos convencionales como decapitaciones, asesinatos masivos y a la detonación de granadas en espacios concurridos para disuadir a la población y a las autoridades de la necesidad de negociar; incluso algunos especialistas de Estados Unidos empezaron a cuestionarse si México se convertiría en un "estado fallido". No obstante, el gobierno mexicano se rehusó a cambiar su principio de confrontación directa en la campaña contra el narcotráfico.

Mientras esto sucedía, desde Estados Unidos se avecinaba una crisis financiera que desaceleraría la economía justo antes de unas elecciones intermedias cruciales para la aprobación de las iniciativas pendientes del Presidente Calderón. Aunque cabe señalar que en los primeros meses de 2008 ya se habían aprobado reformas importantes como la Reforma Judicial, que incluía la creación de juicios orales, el supuesto de inocencia del acusado y otras provisiones para hacer más expedita la administración de justicia en el combate al crimen organizado.

Gracias a estos retos y según datos de la encuesta realizada por GEA-ISA en noviembre de 2008, la popularidad de Calderón entre los votantes ha disminuido de manera constante de marzo de 2007 a noviembre de 2008. El porcentaje de los encuestados que creen que Felipe Calderón tiene mucha capacidad para gobernar ha descendido de 46 al 28 por ciento. Como los efectos de la desaceleración económica se sintieron hasta finales del año, lo más probable es que esto haya sido una reacción a los problemas de seguridad pública. Por ejemplo, los encuestados que dicen que confían en el presidente para resolver problemas cayeron de 36 a 26 por ciento, indicando que para muchos el Ejecutivo se vio rebasado por los problemas principales de la nación. Finalmente, el porcentaje de aquellos que piensan que el presidente respeta la legalidad descendió 15 puntos; es decir, de un 40 a un 25 por ciento. Esto se debe en parte al hecho de que el Ejército está más activo en varios estados de la República, y ha sido blanco de varias acusaciones por parte de grupos de derechos humanos. Las cifras de popularidad son preocupantes para el PAN por la cercanía de las elecciones intermedias que suelen ser consideradas como un referéndum del desempeño del Ejecutivo. No obstante, uno debe comparar estos indicadores con lo que podrían llegar a ser reformas verdaderamente importantes como la del sistema penal, o la educativa como se verán más adelante.

Después de los éxitos legislativos del 2007 (la reforma al sistema de pensiones, al sistema tributario y las consultas para la reforma judicial), el partido en el gobierno creyó que contaba con la inercia suficiente para promover una iniciativa del sector energético. La 
principal reforma del 2008 sería la modificación del régimen de la compañía petrolera estatal Petróleos Mexicanos (PEMEX). Pero la reforma fue considerada como superficial, incluso antes de que Andrés Manuel López Obrador y el Frente Amplio Progresista (FAP) realizaran una larga campaña en su contra. ${ }^{1}$ El petróleo es muy importante para las finanzas del gobierno, pues casi el 40 por ciento de los ingresos del erario provienen de la extracción de hidrocarburos; sin embargo, dicha iniciativa se presentaría en el Congreso hasta finales de abril del 2008. Para avanzar en la reforma energética, el gobierno de Calderón decidió adoptar una estrategia minimalista que le permitiera asegurar los votos del Partido Revolucionario Institucional (PRI) y construir una mayoría legislativa. El PRI nunca ha defendido una reforma sustancial a PEMEX, porque está obligado a conciliar los intereses del partido con el de los sindicatos petroleros (y otros grupos políticamente relevantes).

Los principios más importantes de la reforma energética serían: autonomía presupuestaria y nuevas condiciones para la contratación de servicios de empresas privadas que "incluían incentivos económicos para aquellas empresas que cumplieran con los plazos acordados para la producción y explotación" (Eurasia Group 2008). La reforma enviada por la administración del PAN originalmente consideraba la inversión privada en la construcción de refinerías y el mantenimiento de los oleoductos (actualmente ninguno de los existentes cumple con las condiciones requeridas). El PRI, sin embargo, no permitió la apertura a la inversión privada (nacional o extranjera) en el refinamiento o transporte.

Aún con esta estrategia minimalista de reforma, los partidos de izquierda, y en particular el PT y Convergencia -vinculados al candidato presidencial del PRD, López Obrador-, decidieron que podían ganar una batalla política importante si acusaban al gobierno federal de intentar privatizar PEMEX. Esto constituía una gran ofensa para la población, pues la petrolera es considerada por la mayoría de los mexicanos como un patrimonio de la nación, y una parte importante de su identidad nacional. Como el PRI y el PAN formaron una mayoría legislativa en el Congreso, el PRD y en menor medida el PT y Convergencia (todos miembros del FAP) utilizaron la ya muy conocida estrategia de tomar la tribuna de ambas cámaras para obligar al gobierno a una negociación. La izquierda había aprendido su lección en el 2007 durante la reforma al sistema de pensiones, cuando los otros partidos aprobaron la iniciativa a través del mayoriteo, por lo que decidió que no sucedería lo mismo con la reforma energética. La estrategia funcionó y, para conciliar los intereses de la izquierda, los legisladores del PAN decidieron organizar en el Senado una consulta pública que duraría muchos meses, y que tendría el efecto de retrasar el proceso de reforma y de diluir la fuerza de una iniciativa ya muy debilitada.

La reforma energética, con todas sus limitaciones, fue discutida en dos etapas, la primera, en medio de una caída en la producción de petróleo en México; y la segunda, durante el último semestre de 2008, cuando bajaron los precios del petróleo en el mundo.

1 El FAP es un ala de centro-izquierda conformada por el Partido de la Revolución Democrática (PRD), el Partido Convergencia y el Partido del Trabajo (PT). 
Gráfico 1: producción mensual de petróleo en México, enero a diciembre, 2008 en miles de barriles

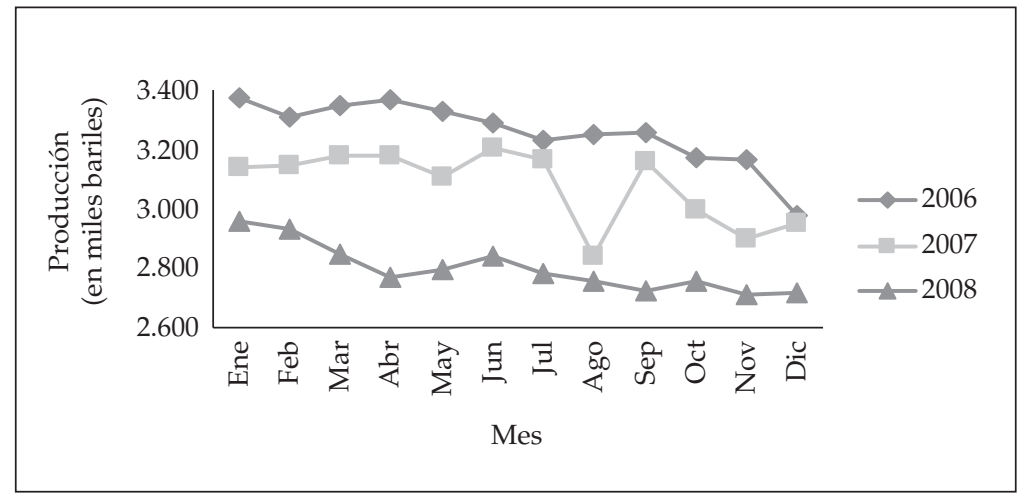

Fuente: Eurasia Group.

La producción de Cantarell, el pozo más importante y prolífico del país, experimenta actualmente un declive, y los pozos secundarios, Ku-Maloob-Zaap, no son lo suficientemente grandes para compensar la producción en el mediano plazo (Eurasia Group 2009).

A finales de octubre, la reforma fue aprobada en ambas cámaras, incluso con votos del ala moderada del PRD. Muchos especialistas consideraron la reforma como demasiado provisional, ya que no incrementaría ni la producción ni resolvería el problema de la ineficiencia o el de la inversión tan escasa (CIDAC, Semana Política, 18 de diciembre, 2008). El gobierno defendió el alcance de la reforma argumentando que ahora PEMEX podría operar de manera más autónoma, y que los nuevos contratos proporcionarían a las empresas privadas los incentivos necesarios para que la producción y la exploración aumentaran (CIDAC, 18 de diciembre de 2008).

Es interesante notar que ni López Obrador ni el ala más radical del FAP fueron capaces de sacar ventaja de la reforma energética, porque ambos se rehusaron a negociar con el gobierno y con la coalición del PRI y el PAN en el Congreso; ni siquiera cuando los miembros más moderados del PRD decidieron participar en el debate (durante la votación definitiva López Obrador protestó en las afueras de la Cámara Alta, obligando a los senadores a reunirse en una sede alterna). Desde finales de abril, López Obrador había recuperado su relevancia política principalmente por su capacidad de frenar el avance de la reforma, pero su negativa a negociar lo mostró como un político subversivo cuyo único interés era ver fracasar al gobierno "espurio" de Felipe Calderón, y no el de mejorar el funcionamiento de la empresa más importante del país.

\section{SITUACIÓN ECONÓMICA}

Por el lado económico, México ha sufrido los efectos del desplome de la bolsa y la crisis del mercado inmobiliario de Estados Unidos. En septiembre, cuando el banco de inversión Lehman Brothers se declaró en quiebra, el valor del peso con respecto al 
dólar empezó a disminuir y, a pesar de los esfuerzos del director del Banco de México y del Secretario de Economía por vender miles de millones de dólares en reservas, poco pudieron hacer para detener su caída.

Gráfico 2: Caída del peso frente al dólar

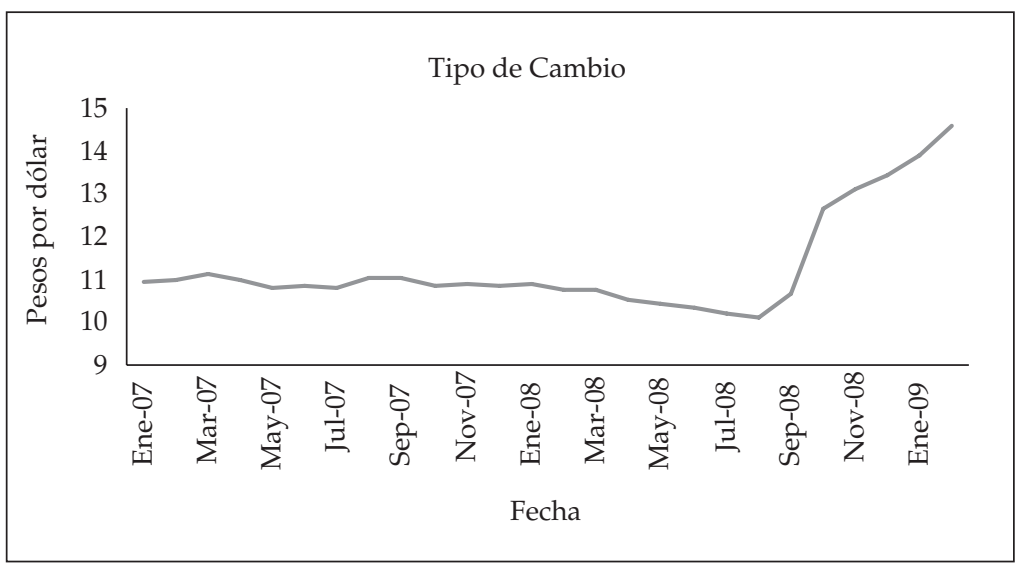

Fuente: Banco de México.

Existen algunas razones que explican la caída del peso mexicano en los últimos meses de 2008, y entre ellas se encuentran el "viaje a la seguridad", la especulación de la moneda, así como el deseo de muchas empresas mexicanas de comprar dólares para liquidar sus deudas, porque tenían la esperanza de que el tipo de cambio se ubicara entre 17-20 pesos por dólar (en septiembre el tipo de cambio era de poco más de 11 pesos por dólar). A pesar de que la economía estadounidense enfrentaba una de las peores crisis económicas de su historia, muchos inversionistas pensaban que los bonos del gobierno de Estados Unidos eran extremadamente seguros (New York Times, 9 de marzo de 2009). La compra de bonos de tesorería por parte de los bancos centrales de otros países y de los inversionistas de Estados Unidos ocasionó que el valor de dólar aumentara con respecto a otras monedas, incluyendo el peso mexicano. Al mismo tiempo, el Secretario de Hacienda, Agustín Carstens, se quejaba abiertamente de que algunas empresas mexicanas estaban apostando en contra del peso, lo que había contribuido a su caída (Agencia EFE, "Ministro de Hacienda de México denuncia operaciones especulativas con el peso", 13 de octubre de 2008).

Ante esta situación, el gobierno mexicano lanzó un paquete anticrisis y subastó miles de millones de dólares para frenar la caída del peso. El programa contracíclico anunciado a principios de octubre incluía ajustes en el precio del petróleo, en el tipo de cambio y en la tasa de crecimiento (de 3 a 1,8 por ciento). El gobierno también se comprometió, por un lado, a destinar más recursos a la infraestructura para disminuir la caída del desempleo y, por el otro, a hacer menos restrictivas las reglas de gasto del presupuesto y a la creación de nuevas políticas que facilitaran su uso (CIDAC, Semana Política, 9 de octubre de 2008). 
La comunidad empresarial mexicana no vio con ningún asombro el programa del gobierno, y criticó lo limitado de sus políticas. Los dirigentes del Consejo Coordinador Empresarial, un grupo de interés que representa a la industria, se quejaron de que el plan no aportaba nada nuevo y que la mayoría de los programas e ideas habían sido anunciados a finales del primer trimestre. Al presidente de la Coparmex, por su parte, le preocupaba que el gobierno hubiera pospuesto los programas de infraestructura, y que no hubiera invertido en el mercado interno (EFINFO, "El plan anticrisis de Calderón es insuficiente, advierte la IP", 9 de octubre de 2008). A finales del año, la bolsa había perdido 35 por ciento de su valor y la segunda fuente más importante de ingresos provenientes del exterior -las remesas- había caído dramáticamente a causa del desempleo de los migrantes en Estados Unidos.

\section{SITUACIÓN POLÍTICA}

Mucho del enredo político del 2008 se explica principalmente por dos razones: La primera fue el deseo del PAN por mantener al PRI como aliado para formar una mayoría en el Congreso que le permitiera aprobar sus reformas pendientes. Y la segunda fue la cercanía de las elecciones intermedias que desató, antes del tiempo de campañas, una contienda entre partidos por mejorar su posición en la Cámara Baja. En dichas elecciones se elegirían a 500 diputados (los senadores se eligen cada seis años junto con el presidente).

Los partidos políticos en México, y en especial los tres más grandes -Partido Acción Nacional (PAN), el Partido de la Revolución Democrática (PRD) y el Partido Revolucionario Institucional (PRI)-, son actores muy poderosos en la política mexicana, pues controlan millones de pesos en recursos públicos, la selección de candidatos y cerca del 90 por ciento de los escaños de ambas cámaras del Congreso. Y gracias a la no reelección consecutiva, los líderes de estos partidos son capaces de mantener la disciplina de los demás legisladores y de centralizar la toma de decisiones. Adicionalmente, ejercen una gran influencia en el funcionamiento del supuestamente autónomo Instituto Federal Electoral (IFE).

Los primeros meses del 2008 fueron testigos de la continuación de un conflicto por ver qué partido del Congreso se haría cargo la dirigencia del IFE, ya que los legisladores no habían sido capaces de llegar a un acuerdo sobre quién reemplazaría al Consejero Presidente (Luis Carlos Ugalde) y a otros dos consejeros, tal como lo marcaba la reforma electoral del 2007. La poca autonomía del IFE frente a los partidos se hizo mucho más evidente después de que estos últimos literalmente fueron capaces de despedir a quien consideraban había actuado de manera desleal (aunque no ilegal). Claramente, los partidos eran los mentores de los consejeros electorales y esta relación continuó en el último proceso de selección, en donde el PRD ganó el derecho de escoger al consejero presidente, el PAN escogió a un consejero, y el PRI al otro. El viejo acuerdo que había existido desde el Consejo de 1997-2003 permitió que los consejeros del IFE gozaran de cierta independencia de sus mentores para que los primeros pudieran decidir con cierta autonomía. Este acuerdo de selección perdió validez desde el proceso de 2003, y 
las nominaciones de 2007-2008 se habían encargado de hacer esto más evidente. Una segunda renovación de los consejeros (establecida también en la reforma electoral de 2007) consolidó la influencia de los partidos sobre "sus" consejeros. Una vez escogidos, numerosos temas formaron parte de la agenda del IFE para el 2008, entre los más importantes se encontraban la preparación del Instituto para sus nuevas tareas del 2009, como el monitoreo de cientos de miles anuncios de campaña en radio y televisión, y la construcción de barreras físicas para evitar que el dinero proveniente del narco llegara a las campañas.

Sin embargo, también los partidos estaban divididos al interior de sus filas, como fue el caso del PRD en el 2008. El PRD se fundó en 1989 a partir de miembros del PRI, líderes de movimientos sociales urbanos y dirigentes de otros partidos de izquierda de los años setenta y ochenta. El PRD siempre se ha caracterizado por ser altamente faccioso y conflictivo en la selección de sus líderes y candidatos. No fue hasta que López Obrador ganó la jefatura del Gobierno del Distrito Federal (GDF) y la candidatura a la presidencia, que comenzó una nueva etapa de hombres fuertes del PRD. López Obrador fue capaz de atraer a las filas del partido a diversos grupos y formar bases electorales sólidas, especialmente entre los votantes que no estaban afiliados a ningún partido político. Sin embargo, después de las elecciones tan cerradas de 2006, el PRD y López Obrador empezaron a perder el apoyo de los votantes, pues su actuación después de los comicios fue percibida como radical.

El PRD tenía programado para el 2008 la elección del nuevo presidente del Comité Ejecutivo Nacional (CEN), y entre los candidatos más fuertes se encontraban, por un lado, Jesús Ortega, dirigente del grupo conocido como los chuchos y, por el otro, Alejandro Encinas, vinculado a López Obrador (quien había colocado a Encinas como jefe de gobierno interino, mientras éste realizaba su campaña presidencial). Jesús Ortega contaba con el apoyo de gran parte de las organizaciones del partido, mientras que Encinas estaba respaldado por las organizaciones informales de López Obrador.

El choque entre ambos grupos de izquierda marcaría durante el resto del sexenio buena parte de las relaciones entre el PRD y el gobierno federal. También era decisivo para el destino del partido en las elecciones intermedias del 2009 y la selección del candidato presidencial en 2012. Con tanta incertidumbre en el resultado -y con un historial de luchas internas por el liderazgo del partido (la elección del CEN de 1999 fue anulada porque los competidores se acusaron mutuamente de fraude)- el PRD tenía que asegurarse que fuera más difícil hacer trampa o cometer algún tipo de fraude. De acuerdo con las reglas de la contienda, sólo los miembros del PRD podían participar, lo que hacía a la a veces variable lista de militantes del partido un tema muy conflictivo.

La votación fue muy cerrada, y hubo resultados muy cuestionables en algunas casillas. Consecuentemente, la dirigencia del partido decidió anular las elecciones. Esta decisión fue apelada por los chuchos, que fueron al Tribunal Federal Electoral (TRIFE) en busca de una decisión final. Mientras esperaban el fallo se nombró a un presidente interino. Finalmente, en octubre de 2008, el TRIFE decidió que, a pesar de haber irregularidades en más del 20 por ciento de las casillas, éstas no eran determinantes para el resultado final, por lo que declaró la anulación improcedente y a Ortega como ganador. 
Ortega es un político moderado que desde su triunfo ha intentado cambiar la relación de su partido con el gobierno federal a través de la negociación en reformas importantes como la reforma energética. López Obrador, por su parte, ha decidido apoyar a sus aliados con candidaturas en el PT y Convergencia, aunque esto no sucede en el D.F. Lo anterior constituye una clara violación a los estatutos del partido, pero Ortega ha decidido no aplicar las sanciones correspondientes porque cree que sólo fortalecerán políticamente a López Obrador.

Gráfico 3: Popularidad de Andrés Manuel López Obrador versus el PRD

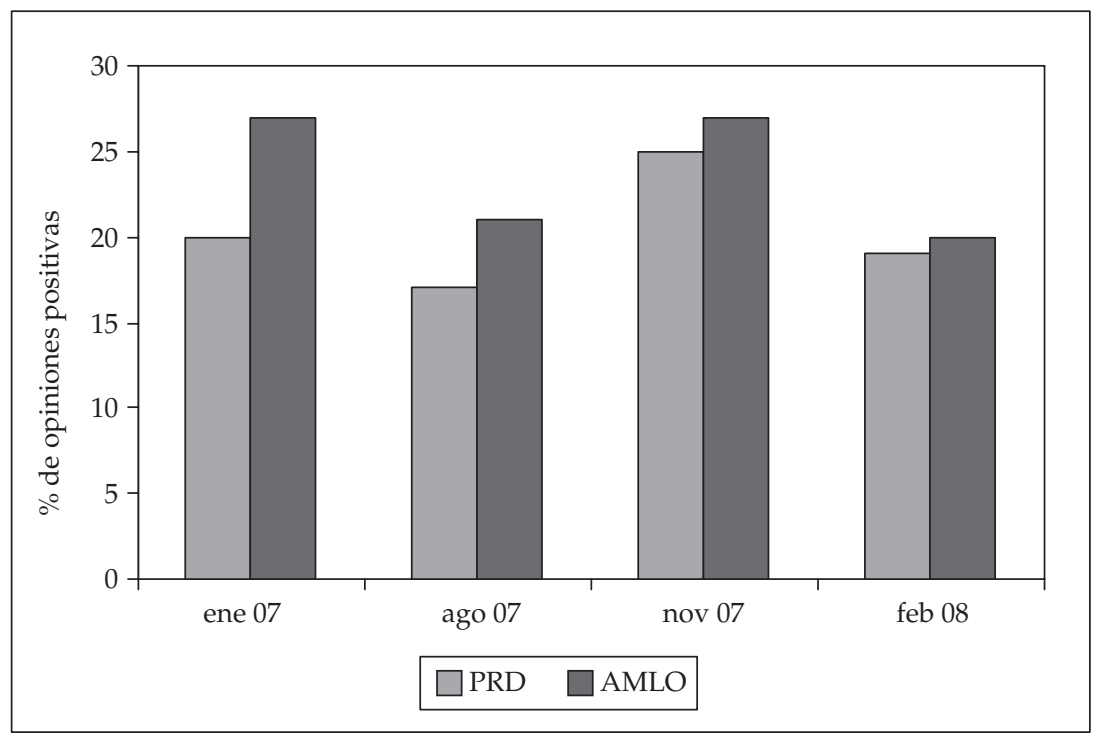

Fuente: www.ipsos-bimsa.com

Gráfico 4: Evolución de la Identidad Partidista, enero 2007 a enero 2009

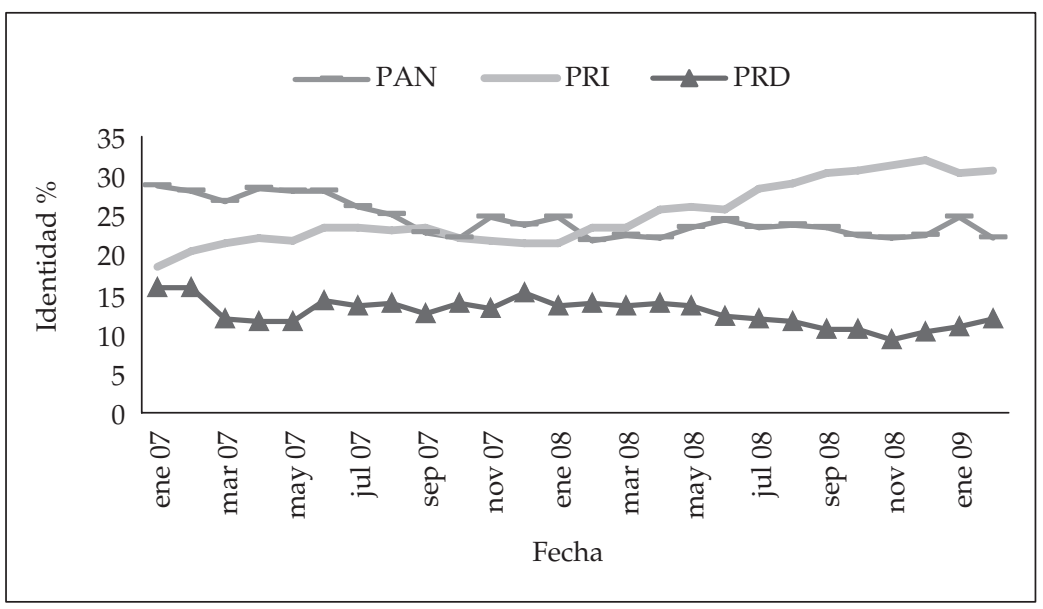

Fuente: Consulta Mitofsky. 
Como muestra la gráfica anterior, desde el inicio de la administración de Calderón en 2007, el PRI se ha vuelto mucho más popular con los votantes, mientras que la identidad del PRD se ha mantenido relativamente estable y se ubica alrededor del 15 por ciento. Este aumento en la popularidad del PRI se debe a varios factores. Primero, la imagen del Presidente Calderón como incapaz de hacer frente contra la amenaza del narco y, en segundo grado, los problemas económicos. En respuesta el PRI ha hecho fiesta de su "experiencia en el poder". Segundo, como se verá posteriormente, el PRI gobierna más estados que los otros dos partidos juntos y estos ejecutivos estatales tienden a gozar de mucha aceptación entre los votantes, lo cual les promueve mayor identificación a todos los políticos de su partido.

\section{Partidos Políticos}

Desde el inicio del sexenio de Fox (2000-2006), la tensión al interior del PAN sobre si el presidente debería privilegiar la lealtad al partido o la lealtad al presidente ha ido en aumento. En los primeros años de la administración el presidente tuvo cierta preferencia por los políticos del PAN de viejo cuño, como el caso del ex gobernador de Jalisco a quien se le concedió como premio la Secretaría de Gobernación. Calderón, por su parte, aprovechó el año nuevo para hacer algunos ajustes a su gabinete que acercarían a sus aliados, como Juan Camilo Mouriño, a portafolios importantes. Como nuevo secretario de Gobernación, Mouriño perdió su capacidad encabezar las negociaciones de la reforma energética cuando el PRD denunció su participación en una serie de contratos entre PEMEX y una empresa de su familia cuando él todavía era funcionario público. Sin embargo, el Presidente Calderón se rehusó a destituirlo. Ernesto Cordero, otro aliado cercano al presidente, fue transferido a la Secretaría de Desarrollo Social (SEDESOL). Tradicionalmente la SEDESOL es una secretaría importante porque controla millones de pesos en recursos para los programas de asistencia social; no obstante, Cordero no ha figurado como un aspirante a la presidencia para el 2012. En noviembre de 2008 muere Mouriño en un accidente aéreo, y Calderón, entonces, decide buscar a alguien fuera de su círculo cercano y nombra a Fernando Gómez-Mont, un experimentado abogado panista, como el nuevo Secretario de Gobernación.

El PAN también destituyó a Santiago Creel - un aliado cercano del Presidente Fox- de su cargo como líder de la fracción parlamentaria del PAN en el Senado, y a Manuel Espino como presidente del CEN del PAN en 2007. Estas acciones han eliminado los vestigios del foxismo en la administración actual y han sido consideradas como una maniobra del partido para mantener su casa en orden.

El PRI, por su parte, ha resurgido desde aquellas elecciones legislativas y presidenciales tan desastrosas del 2006, y ahora es el colaborador clave del PAN para aprobar sus reformas estructurales, aunque en versiones más moderadas. Y a pesar de que el PRI sólo tiene 107 de los 500 escaños, su delegación es crucial para crear mayorías. Además, el PRI continúa con una racha de victorias en las elecciones locales y estatales y se perfila para recuperar algunos escaños en las elecciones intermedias del 2009 (aunque estos resultados tan favorables han disminuido en las encuestas de finales de 2008). 
Actualmente, hay dos candidatos fuertes para la elección interna del PRI del 2012: El gobernador del Estado de México, Enrique Peña Nieto, y el líder de la bancada del PRI en el Senado, Manlio Fabio Beltrones (ex gobernador de Sonora y anterior subsecretario de Gobernación). Peña Nieto es un político mucho más carismático y, gracias al control de las arcas del Estado, se muestra como un candidato generoso con los recursos. Beltrones, por otro lado, es reconocido por ser un líder capaz que piensa de manera estratégica.

Si bien es cierto que los partidos políticos disfrutan de muchas prerrogativas y de un enorme poder, también lo es que los ciudadanos no tienen una opinión positiva de ellos, especialmente si lo comparamos con otras instituciones importantes como el IFE o el Ejército.

Gráfico 5: Calificación de las instituciones enero 2008, diciembre 2008

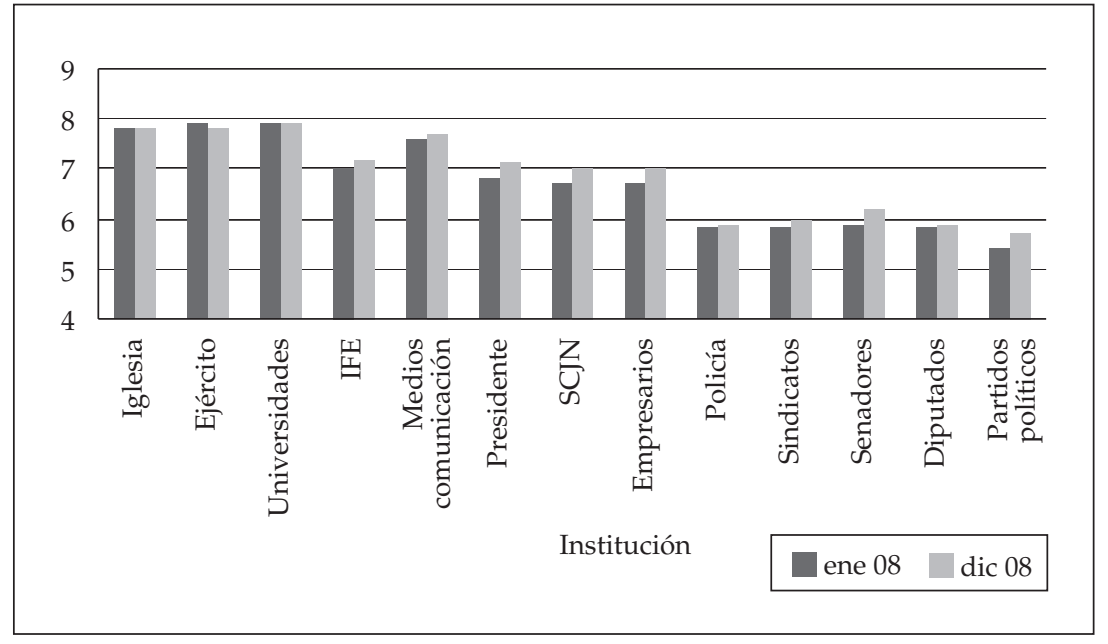

Fuente: Consulta Mitofsky.

Los partidos políticos y los representantes son las instituciones menos respetadas en México, mientras que la Iglesia y el Ejército son los mejor evaluados. Si tomamos en cuenta que el Ejército lleva dos años en las calles luchando en contra de los carteles, una calificación casi de 8 puede ser especialmente importante.

\section{Resultados de elecciones nacionales y subnacionales}

En el 2008 se renovaron únicamente el Congreso y los ayuntamientos de seis estados, dando un total de 381 cargos de elección popular. El principal ganador de este proceso fue el PRI al obtener 208 de estos cargos; mientras que el PRD ganó 91 y el PAN 41. El resto se repartió entre las demás fuerzas políticas. Los tres partidos más importantes compiten de manera independiente únicamente en el estado de Coahuila; en las demás entidades forman coaliciones -para algunos o todos los distritos- con el Partido Verde 
Ecologista de México (PVEM), con el Partido Nueva Alianza (PANAL), o con el Partido del Trabajo (PT) y Convergencia.

De acuerdo con las reglas electorales en México, tanto en las elecciones locales como en las federales, un partido puede obtener escaños por dos vías: mayoría relativa (MR) o representación proporcional (RP). Bajo dichos estatutos, tienen derecho a por lo menos un representante en la Cámara todos aquellos partidos o coaliciones que obtengan en la elección estatal un porcentaje de la votación superior al dos por ciento, y el número de diputados dependerá de los resultados de ésta. Sin embargo ningún partido podrá obtener un número de diputados mayor al número de distritos de la entidad. Los resultados electorales de los congresos estatales son los siguientes:

Baja California Sur (3 de febrero)

\begin{tabular}{lrrccr}
\hline Partido o Coalición & $\begin{array}{c}\text { Núm. } \\
\text { votos }\end{array}$ & Porcentaje & $\begin{array}{c}\text { Escaños } \\
\text { MR }\end{array}$ & $\begin{array}{c}\text { Escaños } \\
\text { RP }\end{array}$ & Porcentaje \\
\hline PAN-PVEM & 32.273 & 19,6 & 0 & 2 & 9,5 \\
PRI & 27.012 & 16,4 & 1 & 1 & 9,5 \\
PRD-PT-Conv & 70.621 & 42,8 & 14 & 0 & 66,7 \\
PMRS & 3.832 & 2,3 & 0 & 1 & 4,8 \\
PANAL & 25.107 & 15,2 & 1 & 1 & 9,5 \\
PAS & 1.011 & 0,6 & 0 & 0 & 0,0 \\
nulos + no reg. & 5.113 & 3,1 & & & \\
Total & 164.969 & 100,0 & 16 & 5 & 100,0 \\
\hline
\end{tabular}

Fuente www.ieebcs.org.mx. www.cbcs.gob.mx

Coahuila (28 de septiembre)

\begin{tabular}{lccccc}
\hline Partido o Coalición & Núm. votos & Porcentaje & $\begin{array}{c}\text { Escaños } \\
\text { MR }\end{array}$ & $\begin{array}{c}\text { Escaños } \\
\text { RP }\end{array}$ & Porcentaje \\
\hline PAN & 128.317 & 17,8 & 0 & 7 & 22,6 \\
PRI & 443.023 & 61,3 & 20 & 0 & 64,5 \\
PRD & 25.744 & 3,6 & 0 & 1 & 3,2 \\
PT & 12.216 & 1,7 & 0 & 0 & 0,0 \\
PVEM & 13.752 & 1,9 & 0 & 0 & 0,0 \\
UDC & 47.689 & 6,6 & 0 & 3 & 9,7 \\
Conv & 3.275 & 0,5 & 0 & 0 & 0,0 \\
PCC & 9.742 & 1,3 & 0 & 0 & 0,0 \\
PANAL & 12.377 & 1,7 & 0 & 0 & 0,0 \\
nulos + no reg. & 26.468 & 3,7 & & & 100,0 \\
Total & 722.603 & 100,0 & 20 & 11 & \\
\hline
\end{tabular}

Fuente: www.iepcc.org.mx/ www.congresocoahuila.gob.mx 
Guerrero (5 de octubre)

\begin{tabular}{lrrrrr}
\hline Partido o Coalición & Núm. votos & Porcentaje & $\begin{array}{r}\text { Escaños } \\
\text { MR }\end{array}$ & $\begin{array}{r}\text { Escaños } \\
\text { RP }\end{array}$ & Porcentaje \\
\hline PAN & 99.538 & 8,8 & 1 & 2 & 6,5 \\
PRI & 324.147 & 28,6 & 11 & 5 & 34,8 \\
PRD & 371.482 & 32,7 & 13 & 6 & 41,3 \\
PT & 23.417 & 2,1 & 0 & 1 & 2,2 \\
PVEM & 22.299 & 2,0 & 0 & 2 & 4,3 \\
Conv & 19.502 & 1,7 & 0 & 1 & 2,2 \\
PANAL & 23.687 & 2,1 & 0 & 1 & 2,2 \\
PSD & 15.430 & 1,4 & 0 & 0 & 0,0 \\
PAG & 12.086 & 1,1 & 0 & 0 & 0,0 \\
PRI-PVEM & 94.489 & 8,3 & 2 & 0 & 4,3 \\
Conv-PT & 84.371 & 7,4 & 1 & 0 & 2,2 \\
nulos + no reg. & 44.613 & 3,9 & & & 0,0 \\
& & & & & 18 \\
Total & 1.135 .061 & 100,0 & 28 & & 100,0 \\
\hline
\end{tabular}

Fuente: www.ieegro.org.mx/www.congrsoguerrero.gob,mx

Hidalgo (17 de febrero)

\begin{tabular}{lrrrrr}
\hline Partido o Coalición & Núm. votos & Porcentaje & $\begin{array}{r}\text { Escaños } \\
\text { MR }\end{array}$ & $\begin{array}{r}\text { Escaños } \\
\text { RP }\end{array}$ & Porcentaje \\
\hline PAN & 81.606 & 12,8 & 0 & 3 & 10,0 \\
PRI & 88.108 & 13,8 & 6 & 1 & 23,3 \\
PRI-PANAL & 226.282 & 35,4 & 12 & 0 & 40,0 \\
PRD & 12.6 .801 & 19,8 & 0 & 2 & 6,7 \\
PT & 16.533 & 2,6 & 0 & 1 & 3,3 \\
PVEM & 28.551 & 4,5 & 0 & 1 & 3,3 \\
Converg & 15.931 & 2,5 & 0 & 1 & 3,3 \\
PSD & 3.775 & 0,6 & 0 & 0 & 0,0 \\
PANAL & 18.190 & 2,8 & 0 & 3 & 10,0 \\
nulos + no reg. & 34.095 & 5,3 & & & 100,0 \\
\hline
\end{tabular}

Fuente: www.ieehgo.org.mx/ www.congreso-hidalgo.gob,mx 
Nayarit (6 de julio)

\begin{tabular}{lccccr}
\hline Partido o Coalición & Núm. votos & Porcentaje & Escaños MR & $\begin{array}{c}\text { Escaños } \\
\text { RP }\end{array}$ & Porcentaje \\
\hline PAN & 65.460 & 17,8 & 1 & 3 & 13,3 \\
PT & 20.212 & 5,5 & 0 & 1 & 3,3 \\
PSD & 4.250 & 1,2 & 0 & 0 & 0,0 \\
PRD-PVEM & 87.125 & 23,7 & 2 & 4 & 20,0 \\
CONV - PRS & 13.005 & 3,5 & 0 & 1 & 3,3 \\
PRI-PANAL & 166.331 & 45,3 & 15 & 3 & 60,0 \\
nulos + no reg. & 11.059 & 3,0 & & & 0,0 \\
Total & 367.442 & 100,0 & 18 & 12 & 100,0 \\
\hline
\end{tabular}

Fuente: www.ieen.org.mx, www.congreso-nayarit.gob.mx

Quintana Roo (3 de febrero)

\begin{tabular}{lrrrrr}
\hline Partido o Coalición & Núm. votos & Porcentaje & Escaños MR & $\begin{array}{c}\text { Escaños } \\
\text { RP }\end{array}$ & Porcentaje \\
\hline PAN & 67.781 & 19,7 & 1 & 3 & 16,0 \\
PRI & 93.918 & 27,3 & 0 & 1 & 4,0 \\
PVEM & 5.160 & 1,5 & 0 & 2 & 8,0 \\
PRI-PVEM & 73.958 & 21,5 & 14 & 0 & 56,0 \\
PRD-PT-CONV & 63.529 & 18,4 & 0 & 3 & 12,0 \\
PANAL & 25.062 & 7,3 & 0 & 1 & 4,0 \\
Alternativa & 3.625 & 1,1 & 0 & 0 & 0,0 \\
nulos + no reg. & 12.604 & 3,7 & & & \\
& & & & 10 & 100,0 \\
Total & 344.594 & 100,0 & 15 & & \\
\hline
\end{tabular}

Fuente: www.ieqroo.org.mx

Estos resultados electorales representan un avance para el PRI con respecto a las elecciones anteriores. En el 2005, el PRI obtiene 94 alcaldías, mientras que para el 2008 gana 22 más. Al mismo tiempo, el número de municipios en manos del PRD y el PAN disminuyen de 67 a 46 y de 27 a 18, respectivamente. La elección de diputados presenta la misma tendencia, a excepción del PAN cuyo número de representantes aumenta ligeramente. Finalmente, las capitales de Guerrero, Nayarit, Hidalgo, Quintana Roo y Coahuila permanecen en manos del PRI, y Baja California Sur del PRD. 
Tabla 2: Puestos elegidos

\begin{tabular}{lrrrrrr}
\hline Partido & \multicolumn{2}{c}{ Alcalde } & \multicolumn{2}{c}{ Diputado Local } & \multicolumn{2}{c}{ Capitales } \\
\hline & 2005 & 2008 & 2005 & 2008 & 2005 & 2008 \\
\hline PRI & 94 & 116 & 85 & 92 & 5 & 5 \\
PRD & 67 & 46 & 55 & 45 & 1 & 1 \\
PAN & 27 & 18 & 21 & 23 & 0 & 0 \\
Otro & 6 & 15 & 25 & 23 & 0 & 0 \\
Total & 194 & 195 & 186 & 183 & 6 & 6 \\
\hline
\end{tabular}

Fuente: www.consultamitofsky.com.mx

Los siguientes cuadros muestran el porcentaje de votos de los partidos en elecciones anteriores. Los años 2003 y 2006 corresponden a las elecciones para diputados federales; los años restantes representan las elecciones locales. Si revisamos los resultados individuales del PAN podemos ver que en ninguno de los estados con elecciones en 2008 obtiene más allá del 20 por ciento. Adicionalmente, hay una caída dramática de la votación en los estados de Coahuila, Hidalgo y Baja California Sur. Y con respecto a las votaciones del 2005 solamente mejora su posición en los estados de Nayarit, Baja California Sur y ligeramente en Guerrero. El PAN no gobierna es estos estados, así que no puede apoyarse en la popularidad del Ejecutivo estatal; además, la popularidad del presidente panista no se ha mantenido muy firme, lo cual también le afecta a nivel estatal.

Tabla 3: Voto para diputados local y federal PAN

\begin{tabular}{lrrrrr}
\hline Estado & 2002 & 2003 & 2005 & 2006 & 2008 \\
\hline Nayarit & 29,5 & 24,6 & 12,0 & 18,3 & 17,8 \\
Coahuila & 29,2 & 35,9 & 35,6 & 39,6 & 17,8 \\
Hidalgo & 14,5 & 23,4 & 14,3 & 24,8 & 12,8 \\
Quintana Roo & 20,9 & 23,9 & 26,1 & 24,8 & 19,7 \\
Guerrero & 8,9 & 6,2 & 8,1 & 13,8 & 8,8 \\
Baja California Sur & 18,0 & 14,9 & 8,9 & 28,8 & 19,6 \\
\hline
\end{tabular}

Fuente: www.consultamitofsky.com.mx. Las elecciones federales son 2003 y 2006.

El PRI, además de obtener la mayor cantidad de votos, presenta un avance consistente en el Estado de Coahuila y Quintana Roo. Su derrota más grande es en Baja California Sur (gobernado por el PRD desde 1998) en donde presenta una caída de casi 20 puntos con respecto a la elección local anterior, y la votación más baja de todas sus elecciones. A pesar de mejorar marginalmente su posición en Guerrero, el PRI gana los cinco municipios más grandes de dicha entidad. 
Tabla 4: Voto para diputados local y federal PRI

\begin{tabular}{llllll}
\hline \multicolumn{1}{c}{ Estado } & 2002 & 2003 & 2005 & 2006 & 2008 \\
\hline Baja California Sur & 24,9 & 30,2 & 36,2 & 20,6 & 16,4 \\
Hidalgo & 55,4 & 47,9 & 55,8 & 34,3 & 49,1 \\
Guerrero & 41,1 & 42,2 & 35,6 & 31,4 & 36,9 \\
Nayarit & 46,9 & 50,4 & 46,9 & 37,8 & 45,3 \\
Quintana Roo & 41,2 & 44,8 & 42,9 & 39,7 & 48,7 \\
Coahuila & 48,6 & 46,9 & 50,6 & 36,3 & 61,3 \\
\hline
\end{tabular}

Fuente: www.consultamitofsky.com.mx. Las elecciones federales son 2003 y 2006.

Aunque el PRD no mejora su votación con respecto a las elecciones anteriores y presenta una caída sustancial en los estados de Quintana Roo, Hidalgo y Guerrero, obtiene la segunda mayor cantidad de votos, y tiene una participación importante en Guerrero y Baja California, estados en donde también cuenta con la gubernatura.

Tabla 5: Voto para diputados local y federal PRI

\begin{tabular}{lccccr}
\hline \multicolumn{1}{c}{ Estado } & 2002 & 2003 & 2005 & 2006 & 2008 \\
\hline Guerrero & 41 & 39,4 & 40,8 & 48,1 & 32,7 \\
Coahuila & 7,4 & 6,3 & 6,7 & 18,3 & 3,6 \\
Hidalgo & 23 & 17,1 & 26 & 32,9 & 19,8 \\
Baja California Sur & 40,8 & 45 & 44,9 & 44,3 & 42,8 \\
Quintana Roo & 15,4 & 8 & 31 & 30,7 & 18,4 \\
Nayarit & 9,6 & 10,6 & 32,3 & 38 & 23,7 \\
\hline
\end{tabular}

Fuente: www.consultamitofsky.com.mx. Las elecciones federales son 2003 y 2006.

Aún con todos los problemas del PRD a nivel nacional, puede apreciarse que el partido de izquierda ha consolidado bloques de votos estables y que ha tenido cierta aceptación en estados donde antes no la tenía. En parte esto se debe a que el candidato del PRD en las elecciones de 2006 logró subir el nivel de apoyo electoral de niveles muy bajos en varios estados.

\section{Poder Legislativo}

El Congreso de México está compuesto principalmente por 3 partidos y en la legislatura actual ocupan alrededor del 90 por ciento de los escaños. Para el PAN y el PRD esto representa un incremento del 40 y 30 por ciento en relación con la legislatura anterior, y una disminución de cerca del 50 para el PRI. En la Cámara de Senadores aparece la misma tendencia sólo que el avance del PAN es mucho más moderado. 
Tabla 6: Integración del Congreso en la LX Legislatura, 2006-2009

\begin{tabular}{lcccc}
\hline \multirow{2}{*}{ Partidos } & \multicolumn{2}{c}{ Cámara de Diputados } & \multicolumn{2}{c}{ Cámara de Senadores } \\
\cline { 2 - 6 } & Escaños & Porcentaje & Escaños & Porcentaje \\
\hline Partido Acción Nacional & 205 & 41 & 52 & 40,6 \\
Partido de la Revolución Democrática & 127 & 25,4 & 26 & 20,3 \\
Partido Revolucionario Institucional & 107 & 21,4 & 32 & 25,0 \\
Partido Verde Ecologista de México & 17 & 3,4 & 6 & 4,7 \\
Convergencia & 17 & 3,4 & 5 & 3,9 \\
Partido del Trabajo & 11 & 2,2 & 5 & 3,9 \\
Partido Nueva Alianza & 9 & 1,8 & 2 & 1,6 \\
Partido Socialdemócrata & 5 & 1 & & \\
Independiente & 2 & 0,4 & & 100 \\
Total & 500 & 100 & 128 & \\
\hline
\end{tabular}

Fuente: http:/ /sil.gobernacion.gob.mx

Desde 1997 ningún partido ha disfrutado de una mayoría en el Congreso, lo que los ha obligado a lograr acuerdos con las demás fuerzas políticas, y son frecuentes las coaliciones de "todos los partidos" (Cásar 2007). Esto también quiere decir que se ha incrementado el número de iniciativas presentadas, la diversidad de las políticas y el grado de conflicto entre los partidos. La siguiente tabla muestra la promoción y aprobación de las iniciativas desde 1985 hasta el 2008. Es decir, desde la última década que el PRI controló la Cámara de Diputados, hasta la década del gobierno dividido.

Tabla 7: Tasa de aprobación legislativa (1985-2008)

\begin{tabular}{lccc}
\hline Legislatura & $\begin{array}{c}\text { Iniciativas } \\
\text { Presentadas }\end{array}$ & $\begin{array}{c}\text { Iniciativas } \\
\text { Aprobadas }\end{array}$ & Porcentaje \\
\hline LIII (1985-1988) & 559 & 223 & 39,89 \\
LIV (1988-1991) & 294 & 131 & 44,56 \\
LV (1991-1994) & 260 & 158 & 60,77 \\
LVI (1994-1997) & 250 & 108 & 43,2 \\
LVII (1997-2000) & 673 & 195 & 28,97 \\
LVIII (2000-2003) & 1.206 & 281 & 23,3 \\
LIX (2003-2006) & 2.800 & 478 & 17,07 \\
LX (2006-2008)* & 2.279 & 285 & 12,51 \\
\hline
\end{tabular}

Fuente: Cásar (2007) y datos del SIL en http:/ / sil.gobernacion.gob.mx

*Hasta el 31 de diciembre de 2008.

Podemos ver que a partir de 1997 el número de iniciativas presentadas se multiplica, pero que el número de iniciativas aprobadas no se incrementa con la misma rapidez. Esto 
puede explicarse por el exceso de carga de trabajo de las comisiones legislativas, y a su diseño tan ineficiente (Lujambio 1995). Actualmente, existen 42 comisiones ordinarias, pero tan sólo cinco de ellas concentran el sesenta por ciento de toda la carga legislativa, y son aquellas que tienen que ver con el sistema tributario, las modificaciones a la Constitución, la gestión entre el Ejecutivo y los distintos grupos sociales y las reformas al código penal. Es decir, tratan los temas más contenciosos de la agenda nacional. El resto de las comisiones dictamina menos de 35 iniciativas por legislatura, y existen por lo menos cinco comisiones que dictaminan menos diez.

Tabla 8: Carga de trabajo de las Comisiones (promedio LVII, LVIII y LIX)

\begin{tabular}{clcc}
\hline Lugar & \multicolumn{1}{c}{ Comisión } & Promedio & Porcentaje \\
\hline 1 & Hacienda y Crédito Público & 358 & 18,8 \\
2 & Puntos Constitucionales & 368 & 16,8 \\
3 & Gobernación & 224 & 11,4 \\
4 & Justicia y Derechos Humanos & 238 & 10,1 \\
5 & Reglamento y Prácticas Parlamentarias & 91 & 4,2 \\
6 & Trabajo y Previsión Social & 82 & 3,9 \\
7 & Salud & 86 & 3,6 \\
8 & Educación Pública y Servicios Educativos & 68 & 3 \\
9 & Seguridad Social & 44 & 2,5 \\
10 & Medio Ambiente y Recursos Naturales & 55 & 2,5 \\
\hline
\end{tabular}

Fuente: Gaceta Parlamentaria http://gaceta.diputados.gob.mx

El incremento en la promoción de iniciativas y el exceso en la carga de trabajo en las comisiones más importantes también se han traducido en una menor tasa de aprobación de las iniciativas del Ejecutivo. En la LVII Legislatura (1997-2000) la tasa de aprobación fue de 84 por ciento; para la LVIII (2000-2003) esta cantidad disminuyó a 78 por ciento; y en la siguiente (2003-2006) bajó hasta 53 por ciento (Cásar 2007). Actualmente, la tasa de aprobación es de alrededor de 64 por ciento (Impacto Legislativo 2009), y aunque es difícil saber si aumentará, es un hecho que el Congreso de México ha asumido un papel de vigilante y de contrapeso de los proyectos del Ejecutivo.

\section{La Reforma Judicial}

A pesar de los fuertes incentivos de los demás partidos por no aprobar reformas que ayuden al PAN en las siguientes elecciones, sí existe un consenso claro sobre la necesidad de una reforma judicial que ya llevaba varios años preparándose. Por desgracia, el sistema penal mexicano es conocido por su falta de transparencia, el poco respeto a los derechos civiles, los jueces corruptos y los tribunales ineficientes. La policía cuenta con pocos elementos para atrapar a los criminales y su entrenamiento suele ser muy pobre. Los Ministerios Públicos (MP) no investigan a fondo (la mayoría de las sentencias 
en el D.F. se aplican a individuos atrapados en flagrancia y por robos cuyo monto no supera los dos mil pesos), y los jueces utilizan procedimientos poco transparentes. De cada 100 crímenes cometidos, 25 son denunciados, y de esos 25, solamente en uno de ellos el acusado termina en la cárcel (Caballero y López Ayllon 2008). Para aquellos que han sido víctimas de algún delito, únicamente el 10 por ciento se toman la molestia de reportarlo a las autoridades.

La reforma judicial discutida en 2007 y aprobada en marzo de 2008 persigue dos objetivos difíciles de conciliar: el primero consiste en reformar radicalmente el sistema de impartición de justicia para cambiarlo de un régimen inquisitivo a uno acusatorio y, el segundo, pretende fortalecer al Estado para hacer frente a la amenaza del narcotráfico ("Destacan los beneficios de la Reforma Judicial", Cuarto Poder, 7 de enero de 2008). El alcance de la reforma es sorprendente: el supuesto de inocencia está claramente plasmado en la Constitución, se crean jueces de control que vigilan los cateos de la policía, existen mecanismos alternativos mucho más flexibles para resolver las controversias y se han delimitado los casos en los que aplica la prisión preventiva para que el acusado pase encarcelado sin sentencia el menor tiempo.

El componente más importante de la reforma judicial es la introducción de los juicios orales, y éstos reemplazarán, en un plazo no mayor a ocho años, a aquellos juicios burocráticos repletos de trámites que toman demasiado tiempo para resarcir a las víctimas. Los juicios orales (acusatorios) incorporan a los jueces, al MP, y a la parte defensora en una audiencia pública donde se presenta y evalúa la evidencia, discuten las partes y se determina la sentencia (Caballero y Ayllon 2008). Bajo el nuevo esquema, el juez ya no tendrá que "investigar" y juzgar; ahora el MP se encargará de la primera, y el juez solamente entregará la sentencia.

La reforma judicial, por otra parte, pretende dotar al Estado de mayores facultades para luchar en contra de los carteles y el crimen organizado; entre ellas, el arraigo. Sin embargo, no se incluyó el derecho de invadir algún domicilio sin una orden del juez aun cuando existiera el supuesto de que la vida de alguien estaba en peligro. El Congreso rechazó esta provisión gracias al cabildeo de los grupos defensores de los derechos humanos (“Anuncian Acciones Vs. Reforma Penal", Infosel, 11 de febrero de 2008).

\section{COYUNTURA INTERNACIONAL: EL PLAN MÉRIDA}

La política del combate al narcotráfico del Presidente Calderón ha traído consigo un incremento en el número de muertes violentas, ejecuciones, tiroteos y secuestros. Las muertes atribuidas al narcotráfico se duplicaron de 2007 a 2008 (ver gráfico siguiente).

El gobierno de Calderón insiste en que estas cifras son el resultado de utilizar al ejército para combatir a los cárteles de la droga, y que hasta el momento esta política ha sido exitosa. Otros se cuestionan si el gobierno mexicano verdaderamente cuenta con la capacidad para hacer frente a los carteles, dados los enormes recursos de estos últimos ("In Drug War, Mexico Fights Cartel and Itself", New York Times, 29 de marzo de 2009; 
Gráfico 6: Muertes violentas 2001-2008

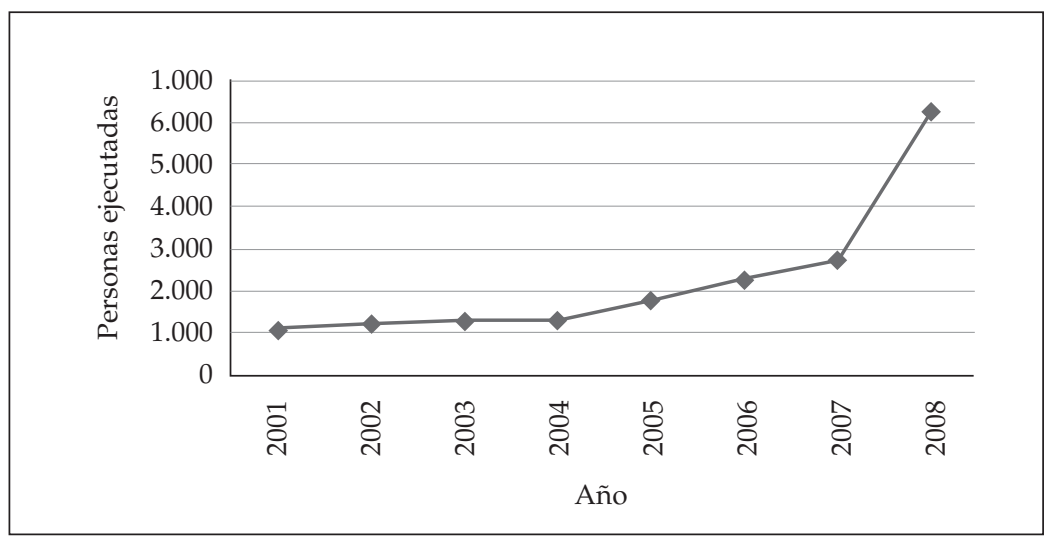

Fuente: Seguridad, Justicia y Paz.

Starr 2009). Además, una vez que capturan o matan a algún líder, éste suele ser remplazado por otros capos de más bajo nivel, lo que desata una lucha territorial entre los mismos narcotraficantes y un aumento en la violencia. Por otra parte, la policía local y estatal -especialmente en aquellas regiones del norte y el centro- suele ser corrupta o inepta.

Un reporte emitido por el United States Joint Forces Command (USJFCOM) a finales de noviembre comparaba a México con Pakistán en el sentido de que ambos eran "[candidatos potenciales de] estados que colapsarían rápida y súbitamente" (2008, 34). El reporte también hacía hincapié en que, si bien era poco probable que México se convirtiera en un estado fallido, "el gobierno, la policía y el sistema de impartición de justicia eran constantemente atacados y presionados por las organizaciones criminales y los cárteles de la droga". Finalmente añadía que si México se volvía un país inestable, sería necesario una respuesta del gobierno de Estados Unidos (aunque el reporte no especifica qué tipo de respuesta) (USFJCOM 2008, 36).

México y Estados Unidos han acordado una iniciativa bilateral -para detener el flujo de drogas de México hacia Estados Unidos- llamada Plan Mérida (por la ciudad en que se celebró dicho acuerdo) (CIDAC, Semana Política, 19 de diciembre de 2007). El Plan Mérida es un paquete de ayuda decretado por el Presidente Bush en el 2008 de 1,4 mil millones de dólares que Estados Unidos destinará a México para un periodo de 3 años. Los fondos serán utilizados para comprar equipo de vigilancia, financiar programas de entrenamiento y adquirir armamento como helicópteros y aviones espía (Washington Post, 5 de abril de 2009). Este paquete sugiere un reconocimiento implícito, por un lado, de que México necesita más recursos y equipo sofisticado para combatir al narcotráfico y, por el otro, de que Estados Unidos también es culpable de la fuerza de los carteles en México, gracias a que Washington ha sido incapaz de controlar la demanda de droga o detener el tráfico de armas de alto poder con las que se abastecen los carteles.

No toda la violencia tiene su origen en el narco. El verano pasado hubo dos casos de secuestro, en donde las víctimas -los dos menores de edad-fueron asesinadas por sus 
captores. Con la creciente ola de secuestros, y en particular del asesinato del hijo de 14 años de uno de los empresarios más acaudalados de México, el gobierno firmó el Acuerdo Nacional por la Seguridad, la Justicia y la Legalidad. Los objetivos principales eran "depurar las instituciones de seguridad pública, fortalecer los mecanismos para combatir el secuestro, regular el uso de celulares para evitar extorsiones telefónicas y consolidar un sistema de información criminal, entre otras medidas" (CIDAC, Semana Política, 5 de febrero de 2009). Sin embargo, el Congreso le dio muy poco seguimiento al Acuerdo, y en una encuesta levantada 150 días después de que el plan se anunció, Consulta Mitofsky encontró que casi la mitad de los encuestados pensaba que la seguridad estaba mucho peor, mientras que poco más del 22 por ciento creía que México era un país más seguro, y otro 22 por ciento nunca antes había escuchado de dicho plan.

Una reforma (que no necesitó la aprobación del gobierno) que puede tener un efecto positivo y duradero en el futuro económico y social de México está relacionada con el decrépito sistema de educación pública. La secretaria de Educación Pública, junto con la lideresa del Sindicato Nacional de Trabajadores de la Educación (SNTE) anunciaron a mediados de 2008 la Alianza por la Calidad de la Educación, y su aportación más importante es la de poner fin a la práctica de los maestros de heredar o vender su plaza. Ahora, los aspirantes al magisterio deben tomar un examen de conocimientos y aquellos con las calificaciones más altas se quedarán en los puestos. Entre simpatizantes del SNTE y sindicatos de maestros de otros estados organizaron una serie de protestas, cancelaron clases y bloquearon las autopistas (estas últimas sucedieron principalmente en Morelos) durante dos meses. Sin embargo, para finales de noviembre los maestros regresaron a sus clases y aparentemente va a salir adelante la reforma para mejorar la educación pública (CIDAC, Semana Política, 11 de noviembre de 2008).

El año 2008 fue difícil para la administración panista, no tanto por los problemas económicos que exportaron los Estados Unidos, sino por la difícil naturaleza de la lucha contra los grandes grupos de crimen organizado que gozan de enormes cantidades de dinero y armas. También, ciertas reformas estructurales han sido tan tímidas en su alcance (como la fiscal de 2007 y la energética de 2008) que no se espera gran resultado de ellas. Peor para el PAN es que los efectos de la deceleración económica ya se están viendo en términos de desempleo y una caída en el crecimiento del PIB, lo cual puede tener consecuencias muy serias para las elecciones intermedias de julio de 2009. El PAN y sus líderes han sido obligados a cambiar su estrategia hacia el PRI, ya que el otrora partido hegemónico ha podido sacar ventaja de los problemas vistos en los primeros años de la administración panista.

\section{REFERENCIAS}

Booth, William y Steve Fainaru, Washington Post, "US Aid Delays in Drug War Criticized", 5 de abril.

Caballero, José Antonio and Sergio López Ayllon. 2008. "Juicios orales, cateos y delincuencia organizada", Nexos 366 (junio): 45-49.

CIDAC, Boletín, Semana Política, varias fechas. [En línea] (http:/ / www.cidac.org/vnm/pdf// / semana_ politica/semana_politica18diciembre2008.html). [Consulta: 16-04-09]. 
Cásar, Ma. Amparo. 2007. Los Gobiernos sin mayoría en México: 1997-2006. Política y Gobierno 15, núm. 1: 221-70.

Eurasia Group. 2008. Mexico: Energy reform on track for November approval. Eurasia Group Report. 14 de octubre.

2009. Mexico: Pemex's oil production decline: No way out. Eurasia Group Report. 21 de enero.

Goodman, Peter S. New York Times. marzo 9 de 2009. "A Rising Dollar Lifts the U.S. but Adds to the Crisis Abroad".

Impacto Legislativo. 2009. "Indicadores de desempeño legislativo". [En línea] (http:/ /impactolegislativo. org.mx). [Consulta: 13-04-09].

Lujambio, Alonso. 1995. Federalismo y Congreso en el Cambio Politico de México. México: UNAM.

Starr, Pamela. 2009. "Mexico and the United States: A Window of Opportunity", Pacific Council on International Policy Special Report". (Los Angeles: Pacific Council on International Policy).

United States Joint Forces Command Center for Joint Futures (USFJCOM). 2008. Joint Operating Environment, (Joint Chiefs of Staff: Norfolk, VA, noviembre ). 


\section{GABINETE PRESIDENCIAL}

\begin{tabular}{lll}
\hline Nombre & Educación & Puesto Anterior \\
\hline
\end{tabular}

\section{Secretaría de Gobernación (SEGOB)}

Juan Camilo Mouriño Terrazo (del 19 de enero de 2008 4, hasta 4 de noviembre de 2008)

Fernando Francisco Gómez-Mont Urueta (desde 10 Licenciatura en derecho de noviembre de 2008)

\section{Secretaría de Relaciones Exteriores (SRE)}

Patricia Espinosa Cantellano

Secretaría de Hacienda y Crédito Público (SHCP)

Agustín Carstens Carstens

\section{Secretaría de la Defensa Nacional (SEDENA)}

Gral. Guillermo Galván Galván

\section{Secretaría de Marina (SEMAR)}

Almir. Mariano Francisco Saynez Mendoza

Gerardo Ruiz Mateos (desde 6 de agosto de 2008)

\section{Secretaría de Desarrollo Social (SEDESOL)}

Beatriz Zavala Peniche

Ernesto Cordero Arroyo (desde el 15 de enero de 2008)

\section{Procuraduría General de la República (PGR)}

Eduardo Medina-Mora Icaza

Secretaría de Seguridad Pública (SSP)

Genaro García Luna

\section{Secretaría de la Función Pública (SFP)}

Salvador Vega Casillas
Licenciatura en economía Jefe de la Oficina de la Presidencia

Abogado del despacho Zinser, Esponda y GómezMont Abogados

Licenciatura en relaciones Embajadora de México en internacionales Austria

Doctorado en economía

Subdirector gerente del Fondo Monetario Internacional

Maestría en seguridad y Subsecretario de defensa defensa nacional

Heroica Escuela Naval Subdirector de Instituto de Militar Seguridad Social para las Fuerzas Armadas Mexicanas ISSFAM

Maestría en economía

Jefe de la Oficina de la Presidencia para las políticas públicas

Ingeniero Industrial

Jefe de la Oficina de la Presidencia de la República

$\begin{array}{ll}\text { Maestría en sociología } & \text { Diputada federal } \\ \text { Maestría en economía } & \text { Subsecretario de Egresos en } \\ & \text { la Secretaría de Hacienda y } \\ & \text { Crédito Público }\end{array}$

Lic. en derecho

Secretario de Seguridad Pública

Ingeniería mecánica

Coordinador del Comité Técnico de la Policía Judicial y/o Ministerial de la Conferencia Nacional de Procuración de Justicia

Maestría en dirección y gestión pública
Subsecretario de la Secretaría de la Función Pública 


\begin{tabular}{|c|c|c|}
\hline Nombre & Educación & Puesto Anterior \\
\hline \multicolumn{3}{|l|}{ Secretaría de Comunicaciones y Transportes (SCT) } \\
\hline Luis Téllez Kuenzler & Doctorado en economía & $\begin{array}{l}\text { Miembro del consejo de } \\
\text { administración de Carlyle } \\
\text { Group }\end{array}$ \\
\hline $\begin{array}{l}\text { Juan Francisco Molinar Horcasitas (desde } 3 \text { de } \\
\text { marzo de 2009) }\end{array}$ & $\begin{array}{l}\text { Maestría en ciencias } \\
\text { políticas }\end{array}$ & $\begin{array}{l}\text { Director general del Instituto } \\
\text { Mexicano del Seguro Social }\end{array}$ \\
\hline \multicolumn{3}{|l|}{ Secretaría del Trabajo y Previsión Social (STPS) } \\
\hline Javier Lozano Alarcón & Lic. en derecho & $\begin{array}{l}\text { Colaborador en la Campaña } \\
\text { de Felipe Calderón }\end{array}$ \\
\hline \multicolumn{3}{|l|}{$\begin{array}{l}\text { Secretaría de Medio Ambiente y Recursos } \\
\text { Naturales (SEMARNAT) }\end{array}$} \\
\hline Juan Rafael Elvira Quesada & $\begin{array}{l}\text { Maestría en ingeniería } \\
\text { agrícola }\end{array}$ & $\begin{array}{l}\text { Subprocurador de } \\
\text { Inspección Industrial en la } \\
\text { Procuraduría Federal de } \\
\text { Protección al Ambiente. }\end{array}$ \\
\hline \multicolumn{3}{|l|}{ Secretaría de Energía (SENER) } \\
\hline Georgina Kessel Martínez & Doctorado en economía & $\begin{array}{l}\text { Directora General de la Casa } \\
\text { de Moneda de México }\end{array}$ \\
\hline \multicolumn{3}{|l|}{$\begin{array}{l}\text { Secretaría de Agricultura, Ganadería, Desarrollo } \\
\text { Rural, Pesca y Alimentación (SAGARPA) }\end{array}$} \\
\hline Alberto Cárdenas Jiménez & $\begin{array}{l}\text { Doctorado en organizació } \\
\text { industrial }\end{array}$ & $\begin{array}{l}\text { Secretario del medio } \\
\text { ambiente y recursos } \\
\text { naturales }\end{array}$ \\
\hline \multicolumn{3}{|l|}{ Secretaría de Educación Pública (SEP) } \\
\hline Josefina Vázquez Mota & Lic. en economía & $\begin{array}{l}\text { Secretaria de Estado en la } \\
\text { secretaría de desarrollo } \\
\text { social }\end{array}$ \\
\hline \multicolumn{3}{|l|}{ Secretaría de Salud (SS) } \\
\hline José Ángel Córdova Villalobos & $\begin{array}{l}\text { Maestría en } \\
\text { administración }\end{array}$ & $\begin{array}{l}\text { Jefe de la División de } \\
\text { Educación Médica del } \\
\text { Hospital Ángeles de León }\end{array}$ \\
\hline \multicolumn{3}{|l|}{ Secretaría de Turismo (SECTUR) } \\
\hline Rodolfo Elizondo Torres & $\begin{array}{l}\text { Licenciado en } \\
\text { administración de } \\
\text { empresas }\end{array}$ & $\begin{array}{l}\text { Secretario de Estado } \\
\text { Secretaría de Turismo }\end{array}$ \\
\hline \multicolumn{3}{|l|}{ Secretaría de la Reforma Agraria (SRA) } \\
\hline Abelardo Escobar Prieto & Ingeniero agrónomo & Diputado federal \\
\hline
\end{tabular}

Fuente:www.presidencia.gob.mx.

Joy Langston Hawkes es Doctora en Ciencia Política por la Universidad de Duke y profesora investigadora del Centro de Investigación y Docencia Económica (CIDE).

[E-mail: joy.langston@cide.edu]

Bernardo Pérez Franco es licenciado en Ciencia Política y Relaciones Internacionales por el CIDE y asistente de investigación en la misma institución.

[E-mail: bernardo.perez@cide.edu] 
